\title{
Article \\ On the Diffuse Interface Models for High Codimension Dispersed Inclusions
}

\author{
Elizaveta Zipunova (D) and Evgeny Savenkov*(D) \\ Keldysh Institute of Applied Mathematics RAS, Miuskaya sq., 4, 125047 Moscow, Russia; zipunova@keldysh.ru \\ * Correspondence: savenkov@keldysh.ru
}

Citation: Zipunova, E.; Savenkov, E. On the Diffuse Interface Models for High Codimension Dispersed Inclusions. Mathematics 2021, 9, 2206. https://doi.org/10.3390/math9182206

Academic Editors: Theodore E. Simos and Charampos Tsitouras

Received: 3 July 2021

Accepted: 5 September 2021

Published: 8 September 2021

Publisher's Note: MDPI stays neutral with regard to jurisdictional claims in published maps and institutional affiliations.

Copyright: (c) 2021 by the authors. Licensee MDPI, Basel, Switzerland. This article is an open access article distributed under the terms and conditions of the Creative Commons Attribution (CC BY) license (https:/ / creativecommons.org/licenses/by/ $4.0 /)$.

\begin{abstract}
Diffuse interface models are widely used to describe the evolution of multi-phase systems of various natures. Dispersed inclusions described by these models are usually three-dimensional (3D) objects characterized by phase field distribution. When employed to describe elastic fracture evolution, the dispersed phase elements are effectively two-dimensional (2D) objects. An example of the model with effectively one-dimensional (1D) dispersed inclusions is a phase field model for electric breakdown in solids. Any diffuse interface field model is defined by an appropriate free energy functional, which depends on a phase field and its derivatives. In this work we show that codimension of the dispersed inclusions significantly restricts the functional dependency of the free energy on the derivatives of the problem state variables. It is shown that to describe codimension 2 diffuse objects, the free energy of the model necessarily depends on higher order derivatives of the phase field or needs an additional smoothness of the solution, i.e., its first derivatives should be integrable with a power greater than two. Numerical experiments are presented to support our theoretical discussion.
\end{abstract}

Keywords: diffuse interface models; phase field model; electric breakdown

\section{Introduction}

Phase field (or order parameter) models form a theoretically sound framework for analysis of a broad class of problems in multiphase hydrodynamics [1], solid mechanics and fracture [2,3], material science, solidification and phase transitions, crystal structures [4-6] and many others research fields. In general, these models are employed to describe dynamics of certain inclusions, i.e., elementary macroscopic constituents (such as droplets), of the dispersed phase immersed into a homogeneous medium. Spatial distribution of the dispersed phase is described by the so-called phase field or order parameter field which is a smooth function of time and spatial coordinates. The phase field function is almost constant inside the regions occupied by the phase and changes rapidly across the inter-phase boundaries. For example, in the context of multiphase hydrodynamics, two or more immiscible fluids are separated by a diffuse interface. The diffuse interface has a certain finite width (hence the term diffuse) which is a model parameter. Therefore, the models have to provide an internal mechanism preventing excessive sharpening or spreading of the diffuse interface during the system evolution.

One of the central concepts of the diffuse interface models is the so called sharp interface limit of the diffuse model which is a model that is raised up when the diffuse interface width tends to zero. In multiphase hydrodynamics a sharp interface limit is usually a free-boundary type model with sharp inter-phase boundaries considered as 2D surfaces, supplemented by certain interface conditions imposed on the conservative variables and their fluxes. A transition from the diffuse interface model to the respective sharp interface one is usually performed using asymptotic analysis and/or the $\Gamma$-convergence framework [3,7-9]. The connection between diffuse interface model and its sharp interface counterpart is rather intimate. On the one hand, for a number of diffuse interface models 
the existence of their physically meaningful sharp interface counterparts gives them a theoretical and practical ground. On the other hand, thermodynamically consistent procedures to derive constitutive relations for phase field models (e.g., using Coleman-Noll procedure [10]) can be applied only if there is an expression for the system energy in a form of a function of the primary thermodynamic variables and their gradients. This function cannot be derived from the thermodynamical considerations alone and has to be somehow postulated. Therefore, it is reasonable to consider the sharp interface models as primary, rather than as derived ones. In many cases the sharp interface models are a source of suitable thermodynamic relations, see, e.g., [11].

In this paper we study a problem with "inclusions" of higher codimension whose evolution is described by the diffuse interface models. For example, in diffuse interface models of multiphase hydrodynamics, the inclusions are just droplets of the dispersed phase. Both continuous and dispersed phases occupy effectively 3D spatial regions which have codimension 0 . The interphase boundary is then a $2 \mathrm{D}$ object with codimension 1 . For diffuse models of fractures the inclusions represent fracture mid-surfaces which have the dimension 2 in 3D settings (and, hence, codimension 1).

To the best of our knowledge, the only example of a phase field model where the inclusions have codimension 2 (i.e., the inclusions are $1 \mathrm{D}$ objects immersed into a 3D medium) is presented in [12]. There, a phase field model for electric breakdown of the dielectric medium is described.

The goal of this paper is to show that codimension of the diffuse inclusion (or, more precisely, codimension of its sharp interface counterpart) significantly restricts the functional dependency of the system energy on derivatives of the state variables. In particular, sticking to the same form of the dependency as in the lower codimension case in models with inclusions of a higher codimension can lead to a mathematically ill-posed problem setting.

Specifically, we show that a model suggested in [12] can not be used to describe electric breakdown channel as an effectively one-dimensional object. Based on certain formal considerations we propose a corrected version of the phase field electric breakdown model.

The model [12] is considered as a specific example of a phase field model of "codimension two". Nevertheless, we believe that the presented considerations are of the general interest and importance and potentially can be applied to the construction of phase field models for a such effectively mixed-dimensional problems as elastic multi-structures [13], dislocation lines dynamics [14], coupled 1D/3D tissue perfusion models [15] and well models in geophysics [16], and numerous electrostatic problems which requires analysis of thin charged or conducting lines, which is a classical problem of electrostatics with numerous application, see, e.g., [17].

\section{Preliminary Discussion}

The diffuse interface model for description of electric breakdown process in solid dielectric medium [12] (see also [18-22]) is constructed as a formal generalization of the well known phase field models for fracture evolution in elastic medium. The derivation of the model is based on the formal analogy between breakdown channel evolution and evolution of the fracture in an elastic medium. The motivation for such generalization is based on the following considerations.

In traditional theories of elastic fracture mechanics [23], the fracture mid-surface is described as a sufficiently smooth two-dimensional surface $\Gamma$ embedded into the three dimensional space-i.e., as a geometrical object of codimension codim $\Gamma=1$. Similarly, electric breakdown channel $\Lambda$ can be considered as a segment of one-dimensional curve embedded into the three-dimensional space-being a geometrical object of codimension $\operatorname{codim} \Lambda=2$.

In both cases, the evolution of codimension 1 fracture mid-surface or codimension 2 breakdown channel is governed by an appropriate internal forces, of the elastic or quasi(electro)static nature, acting in the medium. Besides this, for both fracture surface and breakdown channel, it is possible to construct the so-called J-integral, which describes energy release rate during the fracture $[23,24]$ or channel $[25]$ evolution. 
This formal correspondence between the processes (qualitatively, the only formal difference between them is the codimension) motivates the authors of [12] to extend phase field fracture models to phase field models for breakdown channel evolution.

We now briefly discuss the main steps of derivation of phase field fracture models (see [2] for an overview), following the variational fracture theory, see [9], ([3], Chapter 3) and references therein.

Consider a spatial domain $\Omega \subset \mathbb{R}^{n}$, with $n=1,2,3$, occupied by a physically and geometrically linear homogeneous elastic medium, to which an external surface and volumetric forces are applied. Let $\Gamma \subset \Omega$ be the fracture mid-surface. Within the variational fracture theory (hereinafter we follow $[3,9,26]$ ) the system energy has the form

$$
\mathcal{J}=\mathcal{J}(\boldsymbol{u}, \Gamma)=\int_{\Omega \backslash \Gamma} W(\nabla \otimes \boldsymbol{u}) d \Omega+\kappa \mathcal{H}^{n-1}(\Gamma),
$$

with $u$ being the displacement field, $\mathcal{H}^{n-1}(\Gamma)$ being the $(n-1)$-dimensional Hausdorff measure, $\kappa$ the specific energy attributed to the unit surface element of the fracture mid-surface, and $W$ the elastic potential. The dimension of the Hausdorff measure is essential here: setting it to $n-1$, one explicitly states that fracture is a geometrical object of codimension 1 .

The functional (1) is the departure point for developing the variational fracture theory. It can be shown that, under certain assumptions which are of no importance now, the displacements $u$ of the medium and the trajectory $\Gamma(t)$ of the fracture are minimizers of (1) at each moment of time. In the specified setting the analysis of the problem is rather complicated: to solve it, we have to compute variations of (1) with respect to the fracture mid-surface configuration, that is, with respect to $\Gamma$. For this reason, it is more convenient to approximate the Hausdorff measure in $(1)$ as $[9,26]$

$$
\mathcal{H}^{n-1}(\Gamma) \approx \int_{\Omega} \gamma_{l}(\phi, \nabla \phi) d \Omega, \quad \gamma_{l}(\phi, \nabla \phi)=\frac{1}{2 l} \phi^{2}+\frac{l}{2}\|\nabla \phi\|^{2},
$$

where $\gamma_{l}$ is a volumetric approximation of the surface energy density, $0<l \ll 1$ is a parameter and $\phi$ is the phase field satisfying boundary conditions $\phi=1$ on $\Gamma, \phi \rightarrow 0$ as $\operatorname{dist}(x, \Gamma) \rightarrow+\infty$.

In this case the energy functional (1) is approximated by

$$
\mathcal{J}_{l}(\boldsymbol{u}, \phi, \nabla \phi)=\int_{\Omega}\left((1-\phi)^{2}+\epsilon\right) W(\nabla \otimes \boldsymbol{u}) d \Omega+\int_{\Omega} \kappa \gamma_{l}(\phi, \nabla \phi) d \Omega
$$

In (3), $0<\epsilon \ll 1$ is a small parameter which prevents (3) from degeneracy when $\phi=1$. Usually $\epsilon$ is chosen to be strictly positive, although it is known that, even with vanishing $\epsilon$, the functional (3) is not degenerate [27]. It can be shown that (3) $\Gamma$-converges to the functional (1) as $l \rightarrow 0$.

If $\Gamma$ is prescribed and $u=0$ (i.e., the media is not deformed) then $\phi$ is the minimizer of

$$
\tilde{\mathcal{J}}_{l}=\tilde{\mathcal{J}}_{l}(\phi, \nabla \phi)=\int_{\Omega} \kappa \gamma_{l}(\phi, \nabla \phi) d \Omega
$$

subjected to boundary conditions

$$
\left.\phi\right|_{\Gamma}=1 ; \quad \phi \rightarrow 0, x \rightarrow \infty
$$

The corresponding Euler-Lagrange equations read:

$$
-\Delta \phi+\frac{1}{l^{2}} \phi=0, \quad x \in \Omega \backslash \Omega \text {. }
$$


In 1D case $\left(n=1, \Gamma=\left\{x_{0}=0\right\}\right)$, the solution $\phi(x)=\exp (-|x| / l)$ of (6) decreases exponentially as $\operatorname{dist}(\boldsymbol{x}, \Gamma) \rightarrow+\infty$. In the multidimensional case, this property also holds. Hence, $l$ in (2) can be related to the width of the diffuse fracture.

In the complete formulation of the problem, when $\Gamma(t)$ is not known a priori, Equation (6) includes a source term depending on the elastic energy density of the medium. The points in space at which the value $\phi=1$ are then assumed to be the points of the fracture. Thus, a new fracture surface is formed where, for example, a sufficiently large tensile elastic stress arises.

The expression for $\gamma_{l}$ in (2) is not unique. Nevertheless, definitely, the dependency of $\gamma_{l}$ on $\phi$ and $\nabla \phi$ has to be chosen in such a way that a minimization problem

$$
\tilde{\mathcal{J}}_{l}=\int_{\Omega} \gamma_{l} d \Omega \rightarrow \min ,
$$

accompanied by the boundary conditions (5) is well-posed.

Consider now the diffuse interface model of the breakdown channel presented in [12]. Essentially, the model is constructed as a formal generalization of the diffuse fracture model: it is implicitly assumed that the behavior of the system is described by the energy functional of the form (cf. (1))

$$
\mathcal{J}=\mathcal{J}(\Phi, \Lambda)=\int_{\Omega \backslash \Lambda} W(\nabla \Phi) d \Omega+\kappa \mathcal{H}^{n-2}(\Lambda),
$$

where $\Phi$ is the electric field potential, $\mathcal{H}^{n-2}(\Lambda)$ stands for $(n-2)$-dimensional Hausdorff measure, $\kappa$ is the specific energy assigned to the unit length of the breakdown channel and $W$ is the electrostatic energy. In the 3D case the dimension of the Hausdorff measure is 2 which explicitly says that $\operatorname{dim} \Lambda=1$.

The key point now is the question how $\gamma_{l}$ should be specified so that

$$
\mathcal{H}^{n-2}(\Lambda) \sim \int_{\Omega} \gamma_{l}(\phi, \nabla \phi) d \Omega,
$$

and the respective variational problem (7) is well-posed for $\operatorname{codim} \Lambda=2$ and $\phi$ subjected to BCs (5). The answer to this question essentially depends on the codimension of $\Lambda$. As shown below, the $\gamma_{l}$ as in (2) cannot be used if $\operatorname{codim} \Lambda=2$, i.e., if $\Lambda$ is a curve in a 3D case or a point in a planar case.

\section{Formal Description of the Model}

Let us briefly outline the phase field model proposed in [12]. Consider a bounded domain $\Omega$ occupied at the initial time $t=0$ by a solid dielectric with electric permittivity $\epsilon=\epsilon_{0}$. During the electrical breakdown, a breakdown channel is formed which can be described as a cylindrical domain of a small radius. By analogy with fractures in an elastic medium, the medium in the channel domain is considered to be damaged and therefore has properties different than elsewhere in the domain. Following the diffuse interface method, it is assumed that the damage spatial distribution is described by at least continuous phase field $\phi=\phi(x, t)$ with values within the range $[0,1]$. The value $\phi=0$ corresponds to the damaged phase (the breakdown channel) and $\phi=1$ to the undamaged material. The values $\phi \in(\epsilon, 1-\epsilon), \epsilon \ll 1$ correspond to the diffuse boundary separating the damaged and undamaged phases. Equations defining the evolution of $\phi$ are chosen such that the channel domain is a small tubular neighborhood of a spatial curve which defines the axis (the middle curve) of the breakdown channel. The effective diameter of this tubular neighborhood in an equilibrium state is defined by a model parameter. 
Assuming the breakdown channel is an ideal conductor, electric permittivity in the channel should be infinitely large. In the practical implementations, the permittivity is assumed to have finite, but sufficiently large values,

$$
\epsilon=\epsilon(\phi)(x, t)=\frac{\epsilon_{0}(x)}{f(\phi(x, t))+\delta_{\epsilon}} .
$$

Here $\epsilon_{0}=\epsilon_{0}(x)$ is electric permittivity of the undamaged medium, $f(\phi)=4 \phi^{3}-3 \phi^{4}$ is the so-called interpolation (or degradation) function, and $0<\delta_{\epsilon} \ll 1$ is a parameter which prevents degeneracy of (9) at $\phi=0$. The role of $f$ is the interpolation of the medium properties between the damaged and undamaged phases, see $[3,9,26]$.

The problem is considered in quasi(electro)static setting and, hence, the electric field distribution is potential.

Following the formal analogy with phase field fracture models, the free energy is defined as

$$
\Pi=\int_{\Omega} \pi d \Omega, \quad \pi(\Phi, \phi, \nabla \phi)=-\frac{1}{2} \epsilon(\phi) \nabla \Phi \cdot \nabla \Phi+\mathcal{G} \frac{1-f(\phi)}{l^{2}}+\frac{\mathcal{G}}{4} \nabla \phi \cdot \nabla \phi,
$$

with $\Phi$ being the electric potential, $\mathcal{G}$ being the specific energy per unit length of the channel and $l$ being the effective channel radius. The first term in (10) is the electrostatic energy of the medium. The other terms form a "phase field part" of the energy and are chosen by the authors of [12] formally: They coincide with that for the energy used in the diffuse fracture models. The primary state parameters of the model are $\Phi, \phi$ and $\nabla \phi$.

Equations that describe the system evolution in the non-stationary case are postulated as

$$
\frac{\delta \pi}{\delta \Phi}=0, \quad \frac{1}{m} \frac{\partial \phi}{\partial t}=-\frac{\delta \pi}{\delta \phi},
$$

where $m>0$ is a phenomenological parameter called mobility. In an expanded form Equation (11) read

$$
\begin{gathered}
\nabla \cdot(\epsilon(\phi) \nabla \Phi)=0, \\
\frac{1}{m} \frac{\partial \phi}{\partial t}=\frac{1}{2} \epsilon^{\prime}(\phi) \nabla \Phi \cdot \nabla \Phi+\frac{\mathcal{G}}{l} f^{\prime}(\phi)+\frac{1}{2} \mathcal{G} \Delta \phi,
\end{gathered}
$$

where $(\cdot)^{\prime} \equiv(\cdot)_{\phi}^{\prime}$ and $\epsilon=\epsilon(\phi)$ is defined by (9). Relation (12) is an equation for the electric potential with a dielectric permittivity depending on $\phi$, whereas (13) is an Allen-Cahn type equation which describes the spatial and temporal evolution of $\phi$.

The medium equilibrium state with free energy (10) is defined by imposing vanishing variations of (10) in $\Phi$ and $\phi$. The corresponding Euler-Lagrange equations are (11) with $1 / m \rightarrow 0$ :

$$
\nabla \cdot(\epsilon(\phi) \nabla \Phi)=0, \quad \frac{1}{2} \epsilon^{\prime}(\phi) \nabla \Phi \cdot \nabla \Phi+\frac{\mathcal{G}}{l^{2}} f^{\prime}(\phi)+\frac{\mathcal{G}}{2} \Delta \phi=0 .
$$

To determine the profile of the phase field described by (14), consider an unbounded domain where the breakdown channel axis is a smooth curve $\Lambda$. Assuming the vanishing electric field in the medium, we see that $\phi$ satisfies

$$
\begin{gathered}
\frac{\mathcal{G}}{l^{2}} f^{\prime}(\phi)+\frac{\mathcal{G}}{2} \Delta \phi=0, \quad \mathbb{R}^{3} \backslash \Lambda, \\
\left.\phi\right|_{\Lambda}=0, \quad \phi(x) \rightarrow 1, x \rightarrow \infty .
\end{gathered}
$$


We now introduce cylindrical coordinates $\mathcal{O} r \theta z$, with $r$ being the distance to the axis $\mathcal{O} z$ and $\theta$ being the polar angle. In what follows, we assume $\Lambda=\mathcal{O} z$ and $\phi=\phi(r)$. In [12] it is stated that in this case $\phi$ satisfies

$$
\begin{gathered}
f^{\prime}(\phi)+\frac{l^{2}}{2} \frac{d^{2} \phi}{d r^{2}}=0, \\
\left.\phi\right|_{r=0}=0 ;\left.\quad \phi\right|_{r \rightarrow+\infty} \rightarrow 1 .
\end{gathered}
$$

Integration of (17) leads to

$$
\frac{d \phi}{d r}=\frac{2}{l} \sqrt{1-f(\phi)}
$$

In [12], Equations (17) and (19) are Equations (13) and (14), respectively.

\section{Analysis of the Model}

The model described in the previous section is the only model known to the authors in which the diffuse interface model is used to describe an object of codimension 2 . While the derivation of the model is the "mechanistic" one (i.e., it is based only on analogy), it is relatively common and widely used. Nevertheless, its particular implementation in [12] is not completely correct. More precisely, the form of free energy used to describe a (codimension 1) "diffuse" fracture cannot be used to describe a (codimension 2) "diffuse" breakdown channel. As will be shown below, this is due to the fundamental mathematical properties of the corresponding expression for the free energy.

First, Equation (17), given in ([12], Formula (13)) as an equation describing an axisymmetric distribution of the order parameter, is incorrect. Indeed, consider the primary Equation (15) for the phase field distribution in 3D domain, which contains an infinitely long straight breakdown channel. Recall that the Laplacian in the cylindrical coordinates $\mathcal{O} r \theta z$ is defined as

$$
\Delta \phi=\frac{1}{r} \frac{\partial}{\partial r}\left(r \frac{\partial \phi}{\partial r}\right)+\frac{1}{r^{2}} \frac{\partial^{2} \phi}{\partial \theta^{2}}+\frac{\partial^{2} \phi}{\partial z^{2}}
$$

Since, due to the symmetry assumptions, $\partial \phi / \partial z=\partial \phi / \partial \theta=0$, it follows from (15) and (20) that

$$
\frac{1}{l^{2}} f^{\prime}(\phi)+\frac{1}{2} \frac{1}{r} \frac{\partial}{\partial r}\left(r \frac{\partial \phi}{\partial r}\right)=0 .
$$

As we see, Equation (21) differs from relations (17) and (19), given in [12] as Equations (13) and (14). It is easy to notice that (17) and (19) are correct only for one dimensional planar case and not for the axisymmetric one as it is stated in [12].

This could be seen just as a small technicality if not for the following observation.

To solve one-dimensional Equation (15), we have to take into account boundary conditions (18) for solution values at $r=0$ and $r \rightarrow+\infty$.

It is well known that setting the Diriclet boundary conditions (BCs) at $r=0$ (which is a point set in 2D and line set in 3D) to determine axisymmetric solutions of the second order equation of the type (15) is ill-posed. This is due to the so-called theorems on "removable isolated singularities" in the theory of PDEs. In particular, such a result is well known in the theory of harmonic functions. Simply speaking it states that if a function is bounded and harmonic outside any arbitrarily small neighborhood of the given point, then it can be extended to this point as a harmonic function. This means that the function value can not be defined arbitrarily at a single point.

The Equation (15) considered here is not the Laplace equation, but is a second order semi-linear elliptic equation with the Laplacian as a leading term. For (15) the required result is given in [28,29] and can be formulated as follows (see also [30,31]):

Consider Equation (15) defined in a domain $\Omega \subset \mathbb{R}^{2}$. Let $\phi=\phi(x)$ be its solution in $\Omega \backslash \omega\left(x_{0}\right)$ with $\omega\left(x_{0}\right)$ being an arbitrarily small neighbourhood of the point $x_{0} \in \Omega$. 
Then $x_{0}$ is a removable singularity-that is, $\phi=\phi(x)$ can be extended to $\Omega$ as the solution of (15) iff $\phi$ grows not faster than $\mu(x)=\ln \left(1 /\left\|x-x_{0}\right\|\right)$, i.e., iff $\lim _{x \rightarrow x_{0}} \phi(x) / \mu(x)=0$.

The boundary condition $\phi\left(x_{0}\right)=\phi_{0}$ with finite $\phi_{0}$ at $x_{0}=0$ ensures fulfillment of these conditions. Hence, $\phi(x)$ satisfies Equation (15) in the whole domain $\Omega$ with boundary conditions defined at its outer boundary. For an axisymmetric problem with $\Omega$ being a disk of the given radius centered at $x_{0}=0$ and boundary conditions (18) at $r=R$ or $r \rightarrow+\infty$, such solution is the trivial one, i.e., $\phi(x)=1$.

Hence, since point boundary conditions cannot be set for semilinear second order PDEs of the considered form, the overall setting considered in [12] is not mathematically correct. Thus, the results presented in [12], in particular, Equations (13) and (14) and the solution in Figure 2 in [12], are not correct either: They describe the planar case, which corresponds to the diffuse interface models for fractures. In this case, actually covered in [12], posing boundary conditions for the solution values is possible at $r=0$; the set of points $r=0$ is a line on a plane in the 2D planar case or a plane in space in the 3D setting. In other words, the Equation (15) in this case is considered in the half-space $0<x_{1}<+\infty$, where $x_{1} \equiv r$.

Note that the Equation (21) can be formally approximated by a suitable finite difference scheme for $r \in[0, R]$ with given Dirichlet boundary conditions at $r=0$ and $r=R$. In this case, the formal numerical solution looks similarly to the one shown in Figure 2 in [12]. However, refining the mesh step size exhibits no convergence: the solution asymptotically tends to 1 at each point except at $r=0$. In other words, the derivative of the numerical solution tends to zero at $r=R$ and to infinity at $r=0$, see Section 6 .

It can be imagined that the lack of numerical convergence was not discovered by the authors of [12] due to the fact that authors solved numerically not the "primary" Equation (21), but the Equation (19), which corresponds to the planar, not axisymmetric case.

The above considerations clearly show that the model presented in [12] has to be corrected to be well-posed. The specific way of such correction in suggested in the following section.

\section{A Corrected Model}

Thus, it is mathematically incorrect to pose boundary conditions for equations (15) on a set $\Lambda$ of codimension 2 modeling a breakdown channel. A simple way to see how the things can be corrected is to consider the problem in a variational setting [32].

The stationary solution $(\Phi, \phi)$ of $(12),(13)$ is a pair of functions minimizing the functional (10) for $\phi$ satisfying BCs (16). It is natural to solve the problem for $\phi \in W_{2}^{1}(\Omega)$, where $W_{p}^{q}(\Omega), \Omega \subset \mathbb{R}^{n}$, is the standard Sobolev space (see, e.g., [33,34]). Due to the well-known trace theorems, a trace for functions $v \in W_{2}^{1}(\Omega)$ is defined only for the sufficiently smooth manifolds of codimension 1 (i.e., for the surfaces in 3D). For manifolds of codimension 2, which are of the interest here, a trace for a function in $W_{2}^{1}(\Omega)$ cannot be defined. Let us therefore modify (10) in such a way that it is possible to define BCs on manifolds of codimension 2 for the functions for which free energy functional is bounded.

According to embedding theorems, $W_{p}^{q}(\Omega) \subset C^{r, \alpha}(\Omega)$, where $|\alpha| \leqslant q, n<p q$ and $1 / p-q / n=-(r+\alpha) / n$, and $C^{r, \alpha}(\Omega)$ is the respective Hölder space. In our case $n=3$, and so for $p q>3$ the traces of $\phi \in W_{p}^{q}(\Omega) \subset C(\Omega)$ are correctly defined on manifolds of codimension 2 .

Looking for the minimal possible $p$ and $q$, we see two natural choices, i.e., $\phi \in W_{2}^{q}(\Omega)$ for $q \geq 2$ and $\phi \in W_{p}^{1}(\Omega)$ for $p \geq 4$. Hence, the energy $\pi$ has to include at least the terms $\pi_{q, 2}$ or $\pi_{1, p}$ defined as

$$
\pi_{q, 2}=\int_{\Omega}\left(\Delta^{q / 2} \phi\right)^{2} d \Omega, \quad \pi_{1, p}=\int_{\Omega} \frac{1}{p}\|\nabla \phi\|^{p} d \Omega .
$$

with $q=2 s, s \geq 1$ and $p \geq 4$. 
A formal generalization of (10) which ensures the well-posedness is

$$
\pi=-\frac{1}{2} \epsilon(\phi) \nabla \Phi \cdot \nabla \Phi+\mathcal{G} \frac{1-f(\phi)}{l^{2}}+\frac{\mathcal{G}}{4} \nabla \phi \cdot \nabla \phi+\alpha \frac{\mathcal{G} l^{2}}{8}(\Delta \phi)^{s}+\beta \frac{1}{p} \mathcal{G} l^{p-2}\|\nabla \phi\|^{p},
$$

with natural $s \geq 1$ and $p \geq 4,\|\cdot\|$ being the standard Euclidean norm in $\mathbb{R}^{3}$, and $\alpha \geq 0$, $\beta \geq 0, \alpha+\beta>0$.

In this case first equation in (11) reduces to (12), and second reduces to

$$
\frac{1}{m} \frac{\partial \phi}{\partial t}=\frac{1}{2} \epsilon^{\prime}(\phi) \nabla \Phi \cdot \nabla \Phi+\frac{\mathcal{G}}{l^{2}} f^{\prime}(\phi)+\frac{\mathcal{G}}{2} \Delta \phi-\alpha \frac{\mathcal{G} l^{2}}{4} \Delta^{2} \phi+\beta \mathcal{G} l^{p-2} \nabla \cdot\left(\|\nabla \phi\|^{p-2} \nabla \phi\right) .
$$

A priori, one cannot state which one of the regularizing terms in the expression (22) and (23) is preferable either form thermodynamic or numerical point of views-or both are needed. We only note that $\alpha>0$ and $\beta=0$ leads to the linear biharmonic term in (23) which poses certain problems in numerical approximations. As will be shown below, this makes solution of (23) to be smooth at points of $\Lambda$. The case $\alpha=0, \beta>0$ leads to $p$-Laplacian term in (23) which is nonlinear, but of the second order.

A model equation describing the evolution of $\phi$ in the case $\Phi=0$, then follows from (23):

$$
\frac{1}{m} \frac{\partial \phi}{\partial t}+\alpha \frac{\mathcal{G} l^{2}}{4} \Delta^{2} \phi-\beta \mathcal{G} l^{p-2} \nabla \cdot\left(\|\nabla \phi\|^{p-2} \nabla \phi\right)-\frac{\mathcal{G}}{2} \Delta \phi-\frac{\mathcal{G}}{l^{2}} f^{\prime}(\phi)=0 .
$$

For $\beta=0$ this equation is widely known as the Swift-Hohenberg equation, a key equation in phase-field crystal modeling, see [5,35]. The original model proposed in [5] contains only a biharmonic term. Examples of Swift-Hohenberg type models with both the biharmonic and the $p$-Laplacian terms are given in [36], see also references therein. Note that the Swift-Hohenberg equations are known to be physically justified: the term, proportional to $\Delta^{2} \phi$ in the expression for the energy appears from physical considerations, see, e.g., [35].

In what follows we will need the simplified dimensionless and stationary form of (24). Choosing the spatial scale as $l$ (so, the "diffuse" breakdown channel is of the radius 1 now), it reads:

$$
\alpha \frac{1}{4} \Delta^{2} \phi-\beta \nabla \cdot\left(\|\nabla \phi\|^{p-2} \nabla \phi\right)-\frac{1}{2} \Delta \phi-f^{\prime}(\phi)=0 .
$$

In the realistic setting Equation (24) or (25) are to be solved in the bounded domain $\Omega \backslash$ $\Lambda$ with $\Lambda \subset \Omega$ being a line set which models (predefined) breakdown channel. The appropriate boundary conditions for phase field $\phi$ can be defined as $\phi(x)=0, x \in \Lambda$; and $\phi(x)=0, x \in \partial \Omega$.

An interesting observation is that natural smoothness of the minimizers to (22) allows to consider also the case of co-dimension 3 "inclusions"-i.e., the points in 3D. The authors are not aware of any suitable and meaningful physical or mechanical interpretation of such co-dimension 3 case and consider it as a part of the future work.

Finally, let us note that an example of a high-order diffuse interface model for fractures is given in [26]. The motivation for using such a model is to improve the smoothness properties of the solution and, as a consequence, the computational properties of the isogeometric finite element method used to solve of the problem numerically. Thus, the generalizations made in [26] are not related to the correctness of the diffuse interface model.

Boundary value problems for the polyharmonic equation with boundary conditions defined at manifolds of higher codimension are studied in [33,37-39]. Correctness of boundary value problems for the quasilinear elliptic equation with $p$-Laplacian is considered in [40].

Note that an alternative attitude about the discussed topic is possible within the socalled "set capacity" theory framework. More precisely, one can assume that a manifold of a high codimension admits imposing Diriclet BCs if it has a positive capacity with respect 
to the given functional. In the considered case the capacity is defined by $\gamma_{l}$ and variational problems (7) and (8) accompanied by BCs (5).

\section{Numerical Experiments}

An extensive theoretical and numerical study of (24) or (25) is out of the scope of this paper and can be regarded as a subject of the future work. Nevertheless, in this section we present numerical studies of the simplified $1 \mathrm{~d}$ axi-symmetric and stationary version of (25) to support theoretical discussion presented in the previous section.

We assume the breakdown channel to be aligned with the axis $\mathcal{O} z$ of the cylindrical coordinate system $\mathcal{O} r \theta z$ and $\partial \phi / \partial z=\partial \phi / \partial \theta=0$. Then $\phi=\phi(r), r \in(0, R)$, and (25) reads

$$
\alpha \frac{1}{4} \frac{1}{r} \frac{\partial}{\partial r}\left(r \frac{\partial}{\partial r}\left[\frac{1}{r} \frac{\partial}{\partial r}\left(r \frac{\partial \phi}{\partial r}\right)\right]\right)-\beta \frac{1}{r} \frac{\partial}{\partial r}\left(r\left|\frac{\partial \phi}{\partial r}\right|^{p-2} \frac{\partial \phi}{\partial r}\right)-\frac{1}{2} \frac{1}{r} \frac{\partial}{\partial r}\left(r \frac{\partial \phi}{\partial r}\right)-f^{\prime}(\phi)=0
$$

The accompanying boundary conditions are $\phi=\partial \phi / \partial r=0$ at $r=0, \phi=1, \partial \phi / \partial r=0$ at $r=R$ for $\alpha \neq 0$ and $\phi(0)=0, \phi(R)=1$ for $\alpha=0$.

According to the discussion, the following fundamental properties of $\phi$ are of the interest: (i) $\phi \subset[0,1]$; (ii) $\phi$ is monotonic in $r$; (iii) a reasonable numerical convergence is observed as the computational grid is refined and (iv) phase field values close to zero are concentrated in a sufficiently small neighbourhood of the origin, i.e., $\phi \rightarrow 1, \partial \phi / \partial r \rightarrow 0$ for $r \rightarrow+\infty$. Generally, these properties are not satisfied a priory for an arbitrary choice of parameters in (25), see, e.g., [41].

\subsection{Numerical Method}

A finite difference method is used to solve the problem numerically. Consider a non-uniform computational mesh with $N$ nodes defined in $\Omega=[0, R]: 0=r_{0}<r_{1}<$ $\ldots<r_{N-2}<r_{N-1}=R$. Let $\omega_{i+1 / 2}=\left[r_{i}, r_{i+1}\right]$ be the mesh cells with centers $r_{i+1 / 2}=$ $\left(r_{i}+r_{i+1}\right) / 2, i=\overline{0, N-2}$. The cell centers form a dual mesh with cells $\omega_{i}=\left[r_{i-1 / 2}, r_{i+1 / 2}\right]$, $i=\overline{1, N-1}$. Furthermore, let $\Delta r_{i+1 / 2}=\left|\omega_{i+1 / 2}\right|=r_{i+1}-r_{i}, i=\overline{0, N-2}$ be a mesh step size for the primary mesh, $\Delta r_{i}=\left|\omega_{i}\right|=r_{i+1 / 2}-r_{i-1 / 2}, i=\overline{0, N-2}$ be a mesh step size for the dual mesh.

The values of $\phi$ related to the nodes of primary and dual meshes are denoted as $\phi_{i}$ and $\phi_{i+1 / 2}$, respectively. The corresponding mesh function is then $\phi_{h}=\left(\phi_{0}, \phi_{1}, \ldots, \phi_{N-1}\right)$. Define $\delta_{i+1 / 2}=\left(\phi_{i+1}-\phi_{i}\right) / \Delta r_{i+1 / 2}$.

At the nodes of the primary mesh, the finite-difference approximation of the Equation (26) is defined at the nodes of the primary mesh and read

$$
\alpha \frac{1}{4} \Delta_{h}^{2} \phi_{h}-\beta \Delta_{p, h} \phi_{h}-\Delta_{h} \phi_{h}-f_{i}^{\prime}=0,
$$

with $f_{i}^{\prime}=f^{\prime}\left(\phi_{i}\right)$, the discrete Laplace operator $\Delta_{h} \phi_{h}$ is defined as

$$
\left[\Delta_{h} \phi_{h}\right]_{i}=\frac{1}{r_{i}} \cdot \frac{1}{\Delta r_{i}}\left[r_{i+1 / 2} \delta_{i+1 / 2}-r_{i-1 / 2} \delta_{i-1 / 2}\right], \quad i=\overline{1, N-2}
$$

discrete $p$-Laplacian is defined as

$$
\left[\Delta_{p, h} \phi_{h}\right]_{i}=\frac{1}{r_{i}} \frac{1}{\Delta r_{i}}\left(r_{i+1 / 2}\left|\delta_{i+1 / 2}\right|^{p-2} \delta_{i+1 / 2}-r_{i-1 / 2}\left|\delta_{i-1 / 2}\right|^{p-2} \delta_{i-1 / 2}\right), \quad i=\overline{1, N-2}
$$

and the discrete biharmonic operator $\Delta_{h}^{2} \phi_{h}$ defined as $\left[\Delta_{h}^{2} \phi_{h}\right]_{i}=\left[\left(\Delta_{h} \circ \Delta_{h}\right) \phi_{h}\right]_{i}$ for $i=\overline{2, N-3}$.

Let us denote a finite-difference approximation to $\partial \phi / \partial r$ by $\partial_{h} \phi_{h} / \partial_{h} r$.

For $\alpha \neq 0$ the difference Equation (27) is defined at the nodes $i=\overline{2, N-3}$, i.e., in all primary mesh nodes, except the first two and the last two ones, where boundary conditions 
$\phi_{0}=0,\left[\partial_{h} \phi_{h} / \partial_{h} r\right]_{0}=0$ and $\phi_{N-1}=1,\left[\partial_{h} \phi_{h} / \partial_{h} r\right]_{N-1}=0$ are defined. Here $\left[\partial_{h} \phi_{h} / \partial_{h} r\right]_{0}$ is approximated with a second order consistency as

$$
\left[\frac{\partial_{h} \phi_{h}}{\partial_{h} r}\right]_{0}=-\frac{2 \Delta r_{1 / 2}+\Delta r_{3 / 2}}{\Delta r_{1 / 2}\left(\Delta r_{3 / 2}+\Delta r_{1 / 2}\right)} \phi_{0}+\frac{\Delta r_{1 / 2}+\Delta r_{3 / 2}}{\Delta r_{1 / 2} \Delta r_{3 / 2}} \phi_{1}-\frac{\Delta r_{1 / 2}}{\left(\Delta r_{1 / 2}+\Delta r_{3 / 2}\right) \Delta r_{3 / 2}} \phi_{2} .
$$

The derivative $\left[\partial_{h} \phi / \partial_{h} r\right]_{N-1}$ is approximated in the same way.

For $\alpha=0,(18)$ is discretized at the nodes $i=\overline{1, N-2}$, whereas in the first and the last mesh nodes the boundary conditions $\phi_{0}=0, \phi_{N-1}=1$ are imposed.

The discretized equations are then solved to find the solution nodal values using the Newton method with iterations performed until the residual 2-norm is decreased by a factor $\varepsilon=10^{-6}$.

\subsection{Calculation Results}

In the first series of calculations, a qualitative behavior of the solution of (26) on parameters $\alpha$ and $\beta$ is studied for $(\alpha, \beta) \in\left\{0,10^{-2}, 10^{-1}, 1\right\}^{2}$.

Computational domain is of a radius $R=1$ in dimensionless coordinates. The uniform mesh with $N=100$ nodes is used. In Figure 1, the solutions of the problem are shown. Each row in the figure displays the plots obtained for the same value of $\alpha$, whereas each column contains the plots for the same value of $\beta$. The top left plot in Figure 1 correspond to the incorrect, formal, solution of the difference scheme.

We observe that the numerical solution is monotonic for all parameter values and the properties (i) and (ii) hold. In general this is not obvious: For the generalized FisherKolmogorov equation (such as (26) with $\beta=0$ ) monotonicity and boundness properties of the solution are known to strongly depend on the values of $\alpha$ and the choice of $f$, see, e.g., [41].

Finally, the series of plots shows qualitative dependency of the phase field solution on values of $\alpha$ and $\beta$. The most difference is observed for values $\alpha=0$ and $\alpha \neq 0$. The former case corresponds to the 2nd order semi-linear PDE with solution that has a sharp spike-like singularity at the origin. The latter case corresponds to the 4th order PDE but solution is smooth at the origin. The best choice is a subject of the interplay between less smooth solution and more simple numerical scheme with relatively small computational stencil and smooth solution and more complex numerical schemes with relatively large computational stencils. This issue is a subject of the future research and numerical analysis of realistic 3D settings. The rows 2 to 4 in the Figure 2 yet looks quite similar, demonstrates the level of the relative influence of the parameters on the solution. The similarity of these plots suggest that a robust choice of the formal parameters is possible.

We now show that problem (26) has a solution with an effectively finite support as $R$ increases. A uniform mesh with $N=100$ nodes is used. The parameter values in (26) are chosen to be $\alpha=0.1, \beta=0.01$. In the Figure 2 solutions of (26) for $R=1,5,10$ are shown. The results confirm property (iii): A numerical convergence is observed.

In this series of calculations, a mesh convergence is checked for the problem considered in [12] (Figure 3, left plot), which corresponds to $\alpha=\beta=0$ here, and for the corrected one, introduced in the current work, with $\alpha=0.1, \beta=0.1$ (Figure 3, right plot).

Three meshes are employed: Two uniform meshes with the number of nodes $N=100$ and $N=1000$, and an adaptive mesh with logarithmically distributed nodes. In the last case the nodes are defined as $r_{i}=\delta^{(N-1-i) / N}, i=\overline{0, N-1}$ with $N=100$ and $\delta=10^{-8}$. The computational domain is of the radius $R=1$ in dimensionless coordinates.

As clearly seen in Figure 3 (left), no numerical convergence is observed as the mesh is refined: the solution approaches $\phi=0$ in all points of $\Omega$ except in $r=0$ where $\phi=1$ and $\partial \phi / \partial r \rightarrow \infty$. In contrast the right hand side plot in figure (3) demonstrates a converging solution of (26). 

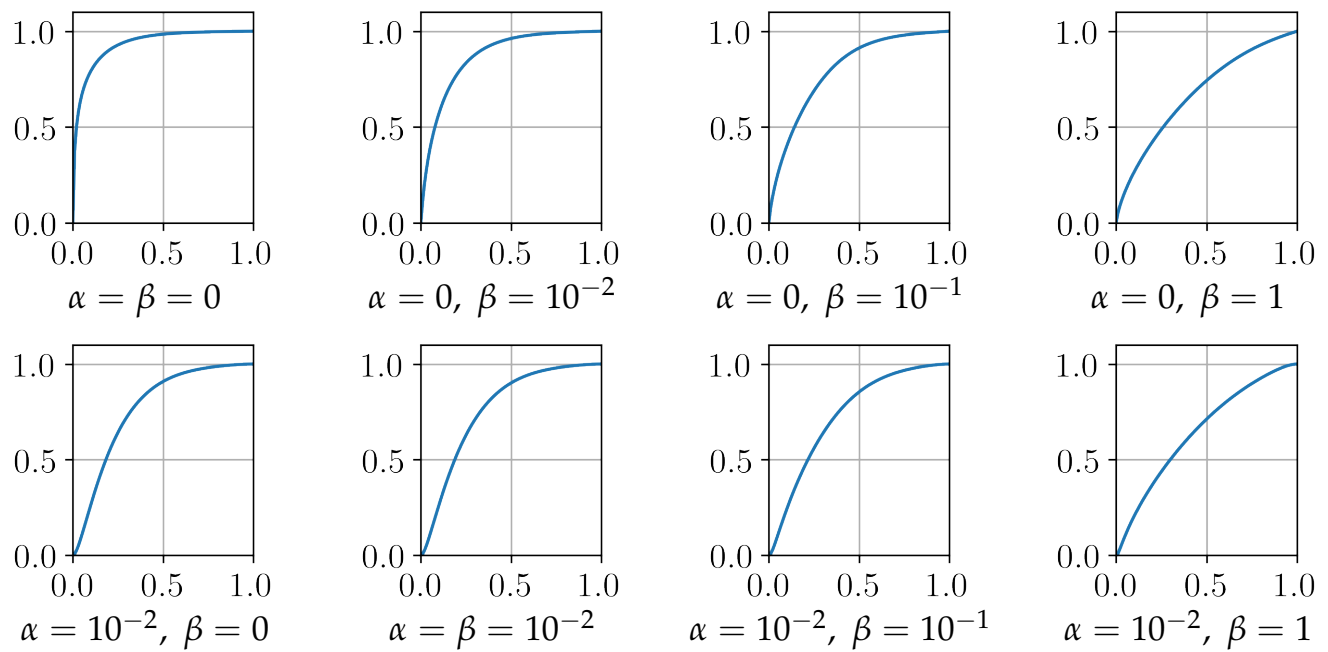

$\alpha=10^{-2}, \beta=0$
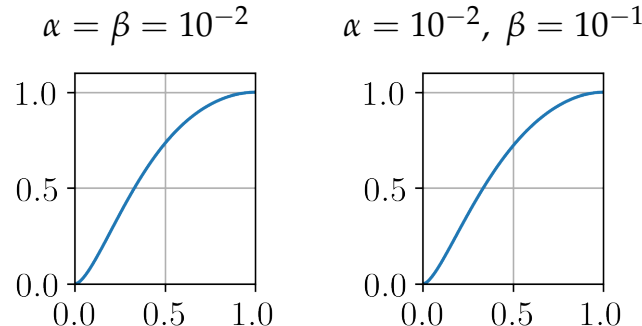

$\alpha=10^{-2}, \beta=1$
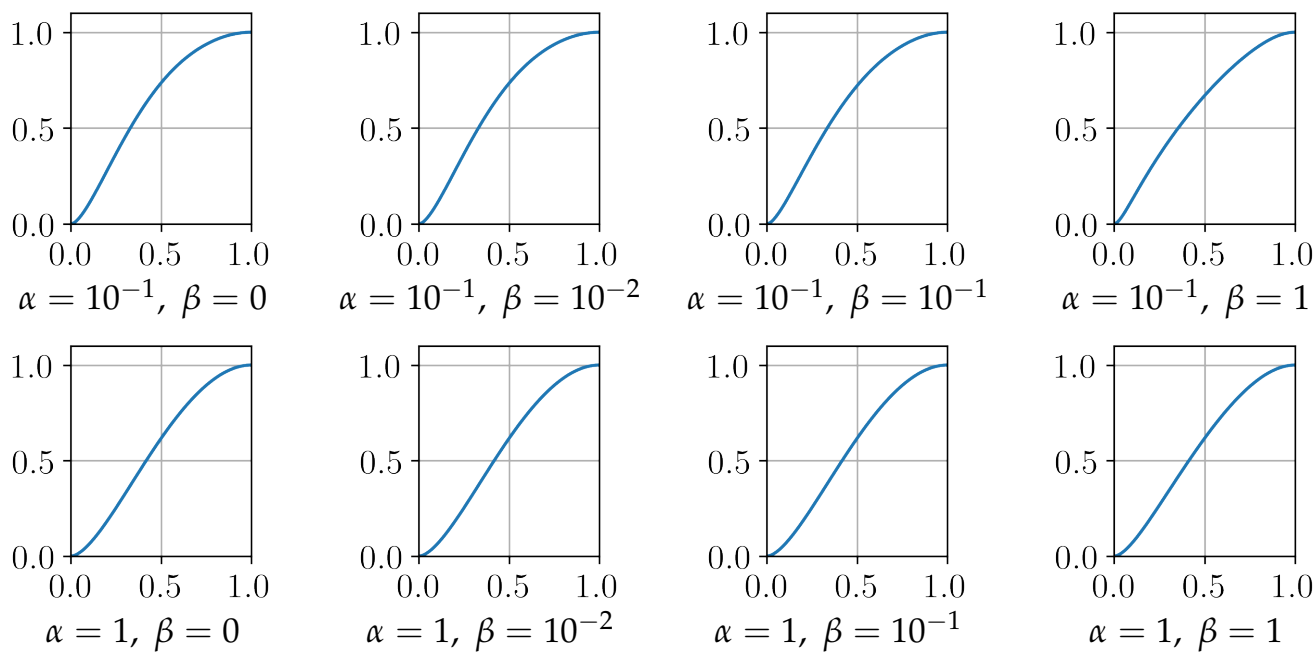

Figure 1. Phase field distribution for $(\alpha, \beta) \in\left\{0,10^{-2}, 10^{-1}, 1\right\}^{2}$ (horizontal axis-polar radius $r$, vertical axis-phase field value $\phi=\phi(r))$.
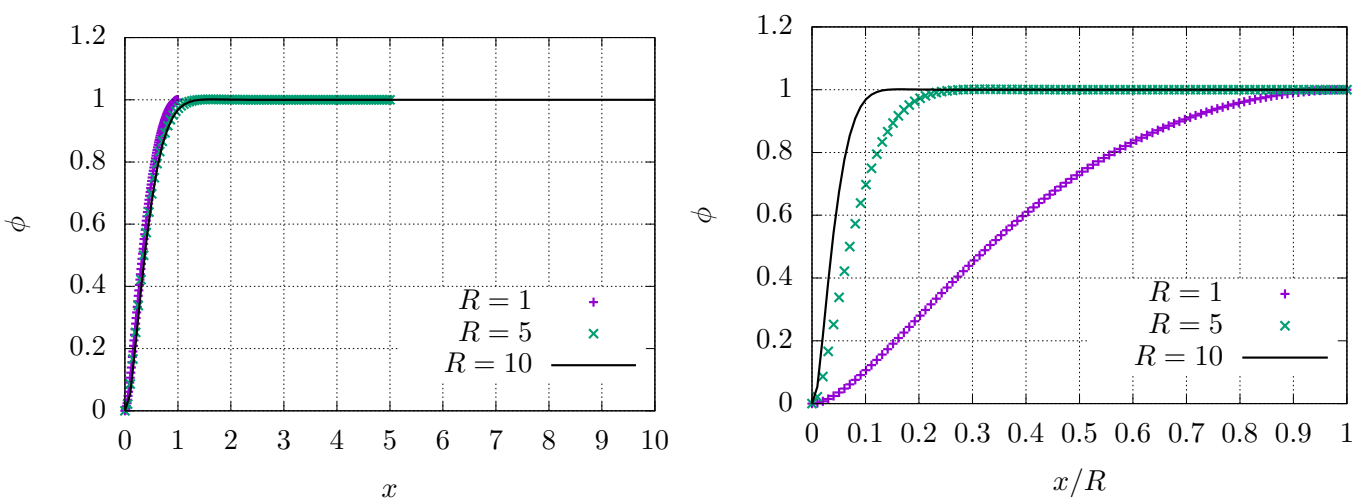

Figure 2. Phase field distribution for $R=1,5,10$ in dimensional (left plot) and dimensionless (right plot) coordinates. 

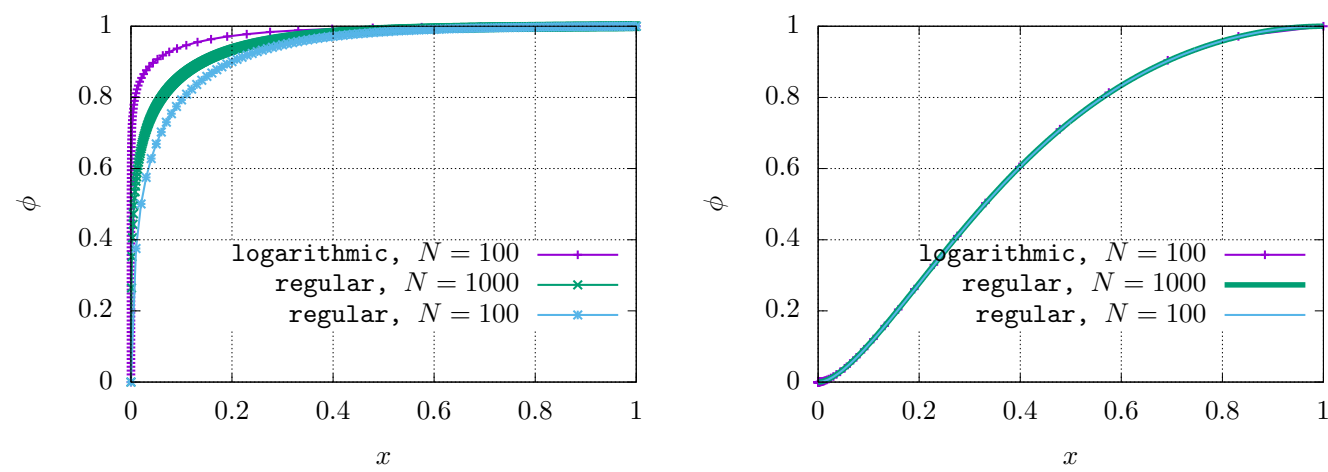

Figure 3. Mesh convergence for $\alpha=\beta=0.0$ (left plot) and $\alpha=0.1, \beta=0.01$ (right plot).

\section{Conclusions}

In this paper we show that the diffuse interface electric breakdown model suggested in [12] is not completely correct from a mathematical viewpoint. More precisely, the model is based on wrong assumptions on the expression for the free energy. The source of the error appears to be a formal generalization of the diffuse fracture models to the case of the (diffuse) breakdown channel. This generalization does not take into account the fact that the co-dimensions of the fracture mid-surface and breakdown channel are different.

Note that the simulation results presented in [12] can be characterized as quite reasonable. However, this does not contradict the theoretical considerations presented by us. In practice, a model inconsistency can often be detected only by studying the numerical convergence of the discrete approximations to the model equations. Such results are not presented in any of the papers cited above, see [12,18-22,42]. Most of these papers explicitly state that the simulations were carried out using a commercial software.

The needed corrections to the model are presented. The corrected form of the free energy is supported by theoretical and numerical arguments. While the particular case of [12] is considered, the authors believe that the obtained results are of the general importance for any phase field models dealing with co-dimension 2 "inclusions".

Finally, probably the main conclusion that can be drawn from this work is that the use of high-order phase-field models ("high" in the sense of "number of derivatives" or in the sense of "power of derivatives") seems to be necessary when a diffuse object is effectively an object of codimension 2 or 3 . While there are papers where high-order diffuse interface models are considered (see, e.g., [26]), up to now their use has not been of fundamental importance and has mainly served for computational efficiency.

Author Contributions: Conceptualization, E.S.; methodology, E.S. and E.Z.; numerical simulations and visualization, E.Z.; writing and editing, E.S. and E.Z.; funding acquisition, E.S. and E.Z. Both authors have read and agreed to the published version of the manuscript.

Funding: This work was supported by the Moscow Center for Fundamental and Applied Mathematics (agreement No. 075-15-2019-1623 with the Ministry of Education and Science of the Russian Federation).

Institutional Review Board Statement: Not applicable.

Informed Consent Statement: Not applicable.

Conflicts of Interest: The authors declare no conflict of interest. 


\section{References}

1. Anderson, D.; McFadden, G.; Wheeler, A. Diffuse-Interface Methods in Fluid Mechanics. Annu. Rev. Fluid Mech. 1997, 30, 139-165. [CrossRef]

2. Ambati, M.; Gerasimov, T.; Lorenzis, L.D. A review on phase-field models of brittle fracture and a new fast hybrid formulation. Comput. Mech. 2015, 55, 383. [CrossRef]

3. Dell'Isola, F.; Gavrilyuk, S.L. Variational Models and Methods in Solid and Fluid Mechanics; Springer: New York, NY, USA, 2011; p. 358.

4. Boettinger, W.J.; Warren, J.A.; Beckermann, C.; Karma, A. Phase field simulation of solidification. Annu. Rev. Mater. Res. 2002, 32, 163. [CrossRef]

5. $\quad$ Elder, K.R.; Katakowski, M.; Haataja, M.; Grant, M. Modeling Elasticity in Crystal Growth. Phys. Rev. Lett. 2002, 88, 245701. [CrossRef] [PubMed]

6. Provatas, N.; Elder, K. Phase-Field Methods in Materials Science and Engineering; Wiley-VCH Verlag GmbH \& Co. KGaA.: Reading, MA, USA, 2010.

7. Abels, H.; Lengeler, D. On sharp interface limits for diffuse interface models for two-phase flows. Interfaces Free Bound. 2014, 16, 395. [CrossRef]

8. Caginalp, G. Phase Field Models and Sharp Interface Limits: Some Differences in Subtle Situations. Rocky Mt. J. Math. 1991, 21, 603. [CrossRef]

9. Bourdin, B.; Francfort, G.; Marigo, J.J. The Variational Approach to Fracture. J. Elast. 2008, 91, 5-148. [CrossRef]

10. Coleman, B.D.; Noll, W. The thermodynamics of elastic materials with heat conduction and viscosity. Arch. Ration. Mech. Anal. 1963, 13, 167-178. [CrossRef]

11. Lowengrub, J.; Truskinovsky, L. Quasi-incompressible Cahn-Hilliard fluids and topological transitions. Proc. R. Soc. Lond. 1998, 454, 2617-2654. [CrossRef]

12. Pitike, K.C.; Hong, W. Phase-field model for dielectric breakdown in solids. J. Appl. Phys. 2014, 115, 044101. [CrossRef]

13. Kozlov, V.; Maz'ya, V.G.; Movchan, A. Asymptotic Analysis of Fields in Multi-Structures; Oxford University Press: Oxford, UK, 1999.

14. Garroni, A.; Marziani, R.; Scala, R. Derivation of a Line-Tension Model for Dislocations from a Nonlinear Three-Dimensional Energy: The Case of Quadratic Growth. SIAM J. Math. Anal. 2021, 53, 4252-4302. [CrossRef]

15. D'Angelo, C. Finite element approximation of elliptic problems with Dirac measure terms in weighted Sobolev spaces: Application to one- and three-dimensional coupled problems. SIAM J. Numer. Anal. 2012, 50, 194-215. [CrossRef]

16. Gjerde, I.G.; Kumar, K. A singularity removal method for coupled 1D-3D flow models. Comput. Geosci. 2020, $24,443-457$. [CrossRef]

17. Cherstvy, A.G. Electrostatics of DNA Complexes with Cationic Lipid Membranes. J. Phys. Chem. 2007, 111, 7914-7927. [CrossRef] [PubMed]

18. Cai, Z.; Wang, X.; Luo, B.; Hong, W.; Wu, L.; Li, L. Nanocomposites with enhanced dielectric permittivity and breakdown strength by microstructure design of nanofillers. Compos. Sci. Technol. 2017, 151, 109-114. [CrossRef]

19. Cai, Z.; Wang, X.; Luo, B.; Li, L. Hierarchical-structured dielectric permittivity and breakdown performances of polymer-ceramic nanocomposites. Ceram. Int. 2018, 44, 843-848. [CrossRef]

20. Cai, Z.; Wang, X.; Li, L.; Hong, W. Electrical treeing: A phase-field model. Extrem. Mech. Lett. 2019, 28, 87-95. [CrossRef]

21. Cai, Z.; Wang, X.; Li, L.; Zhao, P.; Zhu, C.; Li, L. Laminated structure-induced high dielectric strength and energy storage density in dielectric composites. Compos. Sci. Technol. 2019, 173, 61-65. [CrossRef]

22. Cai, Z.; Wang, H.; Zhao, P.; Chen, L.; Hui, C.Z.K.; Li, L.; Wang, X. Significantly enhanced dielectric breakdown strength and energy density of multilayer ceramic capacitors with high efficiency by electrodes structure design. Appl. Phys. Lett. 2019, 115, 023901. [CrossRef]

23. Cherepanov, G.P. The propagation of cracks in a continuous medium. J. Appl. Math. Mech. 1967, 31, 503-512. [CrossRef]

24. Rice, J.R. A Path Independent Integral and the Approximate Analysis of Strain Concentration by Notches and Cracks. J. Appl. Mech. 1968, 35, 379-386. [CrossRef]

25. Suo, Z. Models for breakdown-resistant dielectric and ferroelectric ceramics. J. Mech. Phys. Solids 1993, 41, 1155-1176. [CrossRef]

26. Borden, M.J.; Hughes, T.J.R.; Landis, C.V.; Verhoosel, C.V. A higher-order phase-field model for brittle fracture: Formulation and analysis within the isogeometric analysis framework. Comput. Methods Appl. Mech. Eng. 2014, 273, 100-118. [CrossRef]

27. Braides, A. Approximation of Free-Discontinuity Problems; Springer: Cham, Switzerland, 1998.

28. Cîrstea, F.; Du, Y. Asymptotic behavior of solutions of semilinear elliptic equations near an isolated singularity. J. Funct. Anal. 2007, 250, 317-346. [CrossRef]

29. Véron, L. Weak and strong singularities of nonlinear elliptic equations, in: Nonlinear Functional Analysis and Its Applications, Part 2. Proc. Sympos. Pure Math. 1983, 45, 477-495.

30. Hirata, K. Removable sets for continuous solutions of semilinear elliptic equations. Manuscripta Math. 2011, 135, $245-262$. [CrossRef]

31. Hirata, K.; Ono, T. Removable singularities and singular solutions of semilinear elliptic equations. Nonlinear Anal. 2014, 105, 10-23. [CrossRef] 
32. Badiale, M.; Serra, E. Semilinear Elliptic Equations for Beginners: Existence Results via the Variational Approach; Springer: Cham, Switzerland, 2010; p. 209.

33. Sobolev, S.L. Some Applications of Functional Analysis in Mathematical Physics. Transl. Math. Monogr. 1988, 90, 10-23.

34. Adams, R.; Fournier, J. Sobolev Spaces. In Pure and Applied Mathematics; Elsevier: Amsterdam, The Netherlands, 2003 ; Volume 140.

35. Elder, K.R.; Provatas, N.; Berry, J.; Stefanovic, P.; Grant, M. Phase-field crystal modeling and classical density functional theory of freezing. Phys. Rev. B 2007, 75, 064107. [CrossRef]

36. Golovin, A.A.; Nepomnyashchy, A.A. Disclinations in square and hexagonal patterns. Phys. Rev. E 2003, 67, 056202. [CrossRef] [PubMed]

37. Oleinik, O.A.; Shaposhnikova, T.A. On the Dirichlet problem for the biharmonic equation in a domain, perforated along manifolds of small dimension. Dokl. Math. 1996, 54, 763-766.

38. Oleinik, O.A.; Shaposhnikova, T.A. On the averaging of the biharmonic equation in a domain punctured along manifolds of small dimension. Differ. Uravn. 1996, 32, 830-842.

39. Sternin, B.Y. General boundary-value problems for elliptic equations in a domain bounded by manifolds of various dimensions. Dokl. Akad. Nauk SSSR 1964, 159, 992-994.

40. Lewis, J.; Nyström, K. Quasi-linear PDEs and low-dimensional sets. J. Eur. Math. Soc. 2018, 20, 1689-1746. [CrossRef]

41. Bonheure, D.; Juraj, F.; Alberto, A. Qualitative Properties of Solutions to Mixed-Diffusion Bistable Equations. Calculus of Variations and Partial Differential Equations; Springer: Cham, Switzerland, 2016; Volume 55.

42. Hong, W.; Pitike, K.C. Modeling breakdown-resistant composite dielectrics. Procedia IUTAM 2015, 12, 73-82. [CrossRef] 